\title{
PAPER \\ Gbit-Class Transmission Using SOA Data Rewriter for WDM-PON
}

\author{
Satoshi NARIKAWA $^{\dagger a}$, Hiroaki SANJOH ${ }^{\dagger \dagger}$, Naoya SAKURAI ${ }^{\dagger}$, and Kiyomi KUMOZAKI $^{\dagger}$, Members
}

\begin{abstract}
SUMMARY We describe the transmission characteristics of a wavelength independent wavelength division multiplexing passive optical network (WDM-PON) based on a wavelength channel data rewriter (WCDR). The WCDR is composed of a linear amplifier (LA) and a saturated semiconductor optical amplifier (SOA), and by using the WCDR in optical network units (ONUs), we can erase the downstream signal and modulate the same wavelength channel with the upstream signal. In this paper, we analyze the data rewriting characteristic, the frequency chirp characteristic and the bit error rate (BER) degradation occasioned by the use of saturated SOAs. Furthermore, we report high-speed transmission with power penalty of less than $1 \mathrm{~dB}$ at bit rates of $1.25 \mathrm{Gbit} / \mathrm{s}, 2.5 \mathrm{Gbit} / \mathrm{s}$ and $10 \mathrm{Gbit} / \mathrm{s}$ for downstream signals and $1.25 \mathrm{Gbit} / \mathrm{s}$ for upstream signals after transmission through $40 \mathrm{~km}$ of single-mode fiber.

key words: wavelength division multiplexing, passive optical network, semiconductor optical amplifier, gain saturation
\end{abstract}

\section{Introduction}

A lot of recent research has been devoted to the wavelength division multiplexing passive optical network (WDM-PON) [1]. WDM realizes a high-capacity and transparent access network by allocating wavelengths among users or services. Moreover, PON makes it possible to achieve a low-cost access network because one fiber is shared by multiple users. However, in the WDM-PON system where the wavelengths are allocated to the users, the problem is the high costs created by the demand that each optical network unit (ONU) transmit at the precise wavelength assigned to the ONU.

To solve this problem, several WDM-PON systems with centralized light sources (CLS) have been proposed [2]-[7]. The basic WDM-PON with CLS is shown in Fig. 1. The WDM-PON with CLS locates all the light sources in the optical line terminal (OLT), so no light source is needed in the ONU. From the OLT, plural downstream signals $\lambda_{\mathrm{d} 1}-$ $\lambda_{\mathrm{dn}}$ and plural continuous lightwaves $\lambda_{\mathrm{u} 1}-\lambda_{\mathrm{un}}$ for upstream signals are multiplexed and transmitted to the wavelength router. The wavelength router selects the downstream signal $\lambda_{\mathrm{d}}$ and continuous lightwave $\lambda_{\mathrm{u}}$, and transmits them to the ONU. In the ONU, $\lambda_{\mathrm{d}}$ and $\lambda_{\mathrm{u}}$ are separated, and $\lambda_{\mathrm{d}}$ is received. The ONU then modulates $\lambda_{\mathrm{u}}$ with the upstream signal and returns it to the OLT. Since this architecture dispenses with the control and management of wavelengths at

\footnotetext{
Manuscript received May 11, 2007.

Manuscript revised July 17, 2007.

†The authors are with NTT Access Network Service Systems Laboratories, NTT Corporation, Chiba-shi, 274-0825 Japan.

${ }^{\dagger}$ The author is with NTT Electronics, Naka-shi, 311-0122 Japan.

a) E-mail: narikawa.satoshi@ansl.ntt.co.jp

DOI: 10.1093/ietcom/e91-b.2.399
}

the ONU, we can realize a low-cost WDM access network.

In this paper, we propose a WDM-PON access system with CLS that uses a saturated semiconductor optical amplifier (SOA) data rewriter. Several transmission characteristics of this configuration are elucidated by a theoretical analysis and experiments. Section 2 describes the conventional WDM-PON with CLS and its problems. Section 3 explains the system configuration of the proposed WDMPON access system with CLS that uses a saturated SOA data rewriter. Section 4 describes the theoretical analysis and shows the calculated results obtained using remaining intensity noise, frequency chirp and SOA frequency response as parameters. In Sect. 5, we demonstrate the bit error rate (BER) of the proposed WDM-PON access system, which uses an optimum SOA that is easy to saturate. Our conclusions are given in Sect. 6.

\section{WDM-PON with Centralized Light Sources}

To realize the wavelength independent ONU, several configurations have been proposed based on the WDM-PON with CLS. There are three main approaches; namely i) using the same wavelength for up- and downstream signals with time division multiplexing (TDM) [2], ii) using different wavelengths for up- and downstream signals with frequency division multiplexing (FDM) [3], [4], and iii) using the same wavelength for up- and downstream signals but different modulation formats [5]-[7].

In the TDM system, the downstream signal carries unmodulated optical packets for the upstream signal. After transmitting the downstream signal, the ONU modulates the unmodulated packets and sends them back to the OLT. However, although this method can realize high spectral efficiency since the same wavelength is used for up- and downstream signals, the bitrate is limited because the unmodulated time slots and the guard band between up- and downstream signals are needed in the downstream signal.

In the FDM system, the OLT supplies continuous lightwaves separate from the downstream signals. The ONU modulates the continuous lightwave and sends it back to the OLT while receiving the downstream signal. Because each signal uses different wavelengths, full-duplex transmission can be realized, which makes it possible to increase the transmission capacity. Unfortunately, this system cannot use the wavelength resources effectively.

Two different systems have been proposed for imposing different modulation formats on the up- and downstream 


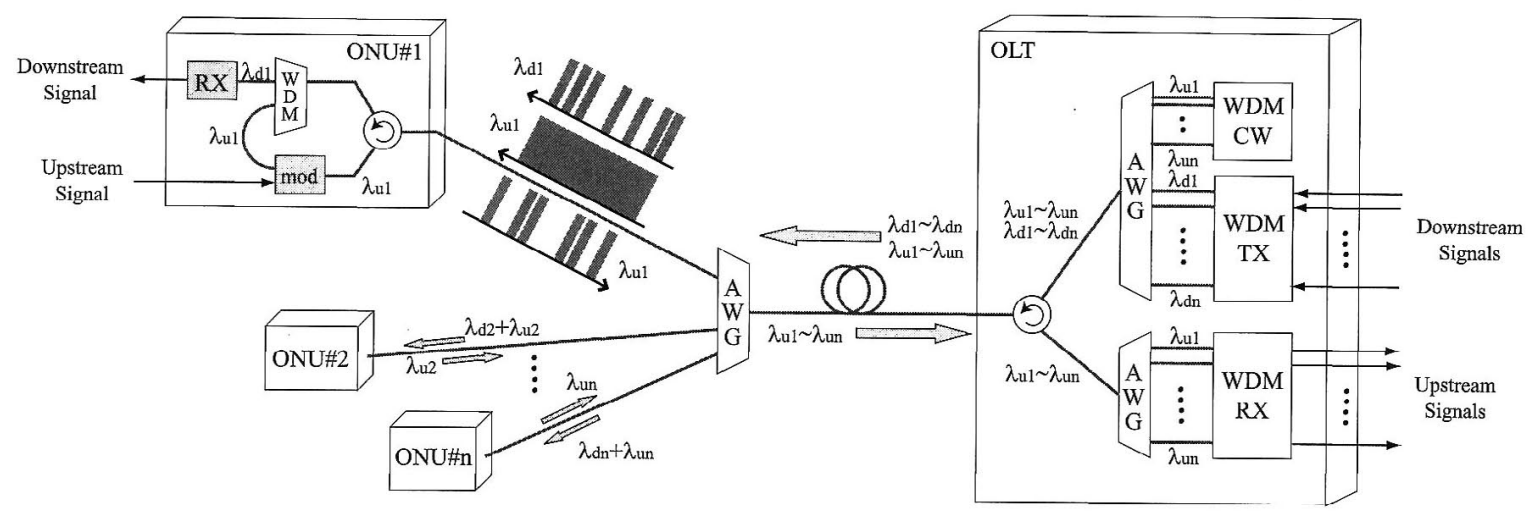

Fig. 1 Schematic diagram of WDM-PON with centralized light sources.

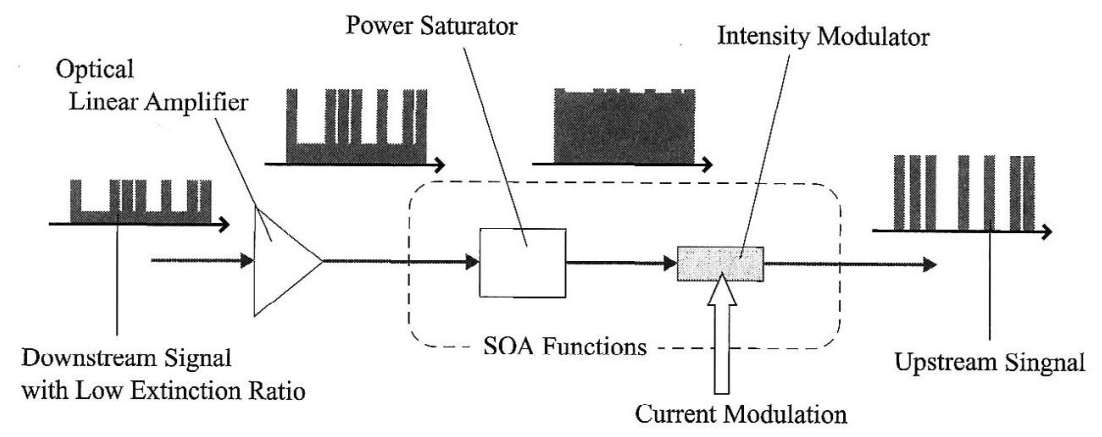

Fig. 2 Principle of wavelength channel data rewriter.

signals. One uses phase or frequency modulation for the downstream signal and intensity modulation for the upstream signal [5], [6]. The other uses inverse return to zero (RZ) intensity modulation for the downstream signal and non-return to zero (NRZ) intensity modulation for the upstream signal [7]. These methods use the same wavelength and do not need to utilize separate time slots, so their wavelength efficiency and transmission capacity are high, but the structure of the receiver is more complex than an ONU that uses NRZ intensity modulation for bidirectional transmission.

Our proposal is a cost-effective and high-capacity WDM-PON with a wavelength channel data rewriter (WCDR) that uses a saturated SOA [8]. It is described in the next section.

\section{Wavelength Channel Data Rewriter Using Satu- rated SOA}

Figure 2 shows the configuration of the proposed WCDR with a saturated SOA. The WCDR is composed of one linear amplifier (LA) and one SOA. First, we use an intensity modulated signal with a low extinction ratio (ER) as the downstream signal, and amplify it to the saturation limit of the SOA with the LA. Next, by inputting the LA output to the saturated SOA, the on-off ratio between the mark level and space level is reduced, so the downstream signal is erased and changed to a continuous lightwave. Finally, we can transmit the upstream signal to the OLT by modulating

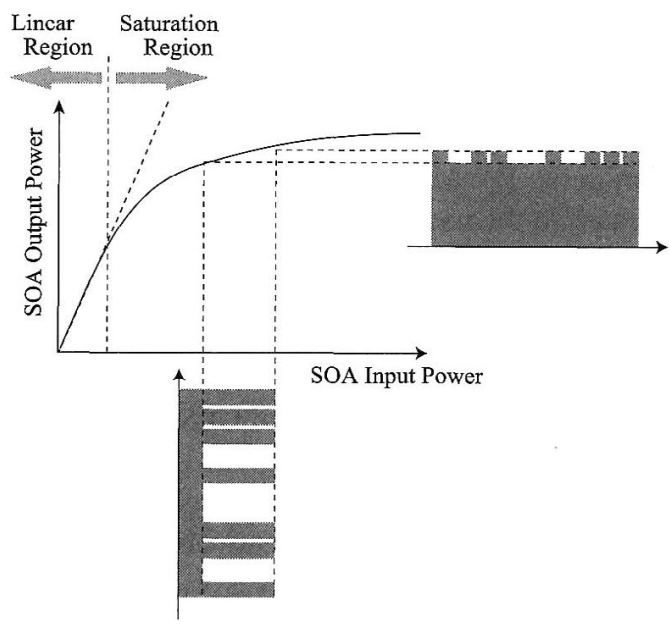

Fig.3 Principle of SOA saturation and data erasing.

the injection current of the SOA. Figure 3 shows the mechanism of optical signal erasing. This figure shows that by using the saturation region of the SOA and inputting the NRZ optical signal with a low ER into the SOA, it is possiblc to erase the input signal modulation and generate a new optical signal without requiring a laser diode with an assigned wavelength.

The WDM-PON with WCDR is shown in Fig. 4. Here, WDM signals are transmitted as the downstream signals from the OLT; the wavelength channels are routed by wavelength router at the PON separation point. In the ONU, one 


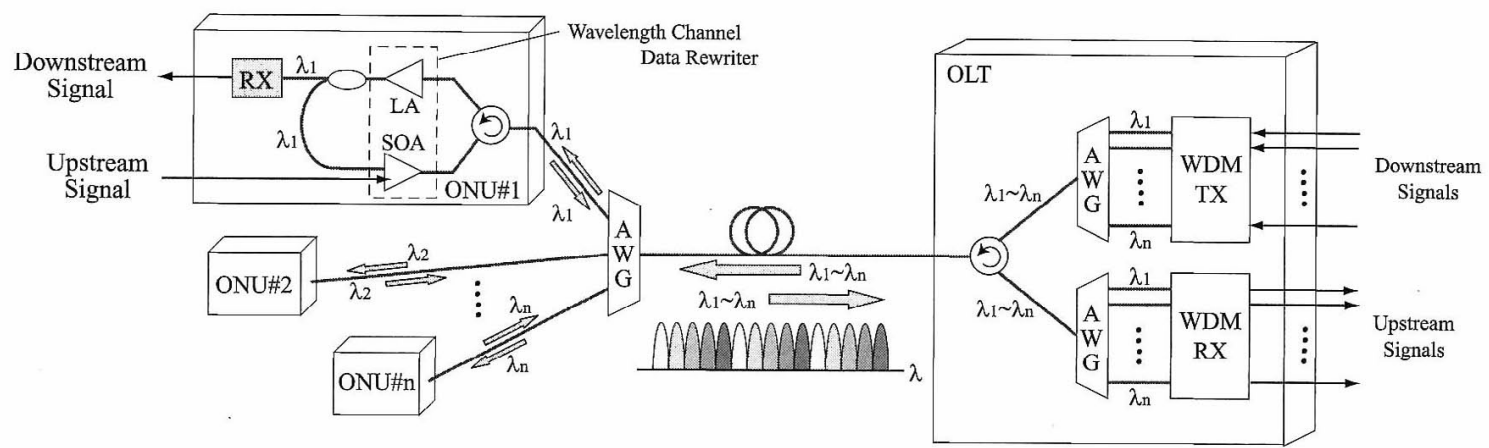

Fig. 4 Schematic diagram of WDM-PON using wavelength channel data rewriter.

part of the downstream signal is extracted, amplified, and received. The remaining part is processed in the WCDR and transmitted to the OLT as the upstream signal. By using this architecture, we can efficiently utilize wavelength resources because the bidirectional signals use the same wavelength, and full-duplex transmission communication is established without the guard time of TDM.

\section{Calculation of Transmission Characteristic}

\subsection{Limitation Factor Contributing to Receiver Sensitivity}

In general, a saturated SOA cannot completely erase the bit pattern of the downstream signal; the SOA output exhibits some remaining intensity modulation because of its slight input power dependence even in a saturated condition [8]. This remaining intensity modulation causes mark level fluctuation in the generated upstream signal and reduces the receiver sensitivity because of eye pattern closure. There are several approaches to minimizing the performance degradation of the upstream signal caused by the remaining downstream signal pattern. One is to reduce the downstream signal ER, another is to increase the SOA input power of the optical signal, and yet another is to increase the SOA injection current. Using these techniques improves the saturation characteristic and the remaining bit pattern is suppressed. Another approach is to increase the modulation index of the SOA's driving current to increase the upstream signal ER. An increase in the upstream signal ER suppresses the remaining downstream signal. By using these methods, we can minimize the performance degradation.

Additionally, optical frequency chirping is triggered when an intensity modulated optical signal propagates through the SOA. In the proposed system, frequency chirp occurs while the downstream data is being erased when the downstream signal is amplified to the saturation region and passed through the SOA (the erasing process). Frequency chirp is also triggered by fluctuations in the refractive index in the active layer caused by changes in the carrier density when modulating the SOA injection current to generate the new upstream signal (= the modulating process).

Moreover, with transmissions of more than several Gbit/s, the receiver sensitivity is degraded caused by the lim- ited frequency response of the SOA.

This study analyzes the effect of the remaining downstream signal, the transmission characteristic caused by the two types of the chirp, and the effect of the SOA frequency response.

\subsection{Basic Calculation}

In this subsection, we describe a calculation method that allows us to analyze the effect of the remaining downstream signal and frequency chirp in the proposed system, we describe a calculation method in this subsection.

The carrier density in the SOA is defined by the following rate equation [9], [10];

$$
\frac{\partial N}{\partial t}=\frac{I}{q V}-\frac{N}{\tau_{c}}-\frac{g(N)}{\hbar \omega_{0}}|A|^{2}
$$

where $N$ is the carrier density, $I$ is the injection current, $q$ is the electron charge, $V$ is the active volume, $\tau_{c}$ is the spontaneous carrier lifetime, $g$ is the gain, $\hbar \omega_{0}$ is the carrier density required for transparency, and $A$ is the optical field.

The optical field can be separated into the amplitude $P(z, \tau)$ and the phase $\phi(z, \tau)$ and is expressed as

$$
A=\sqrt{P} \exp (i \phi)
$$

$P$ and $\phi$ are obtained from the following equations;

$$
\begin{aligned}
& \frac{\partial P}{\partial z}=\left(g-\alpha_{i n t}\right) P \\
& \frac{\partial \phi}{\partial z}=-\frac{1}{2} \alpha g
\end{aligned}
$$

where $g$ is the SOA gain, $\alpha_{i n t}$ is the internal loss, and $\alpha$ is the linewidth enhancement factor [11], [12]. From Eqs. (3) and (4), the relation between the input and output optical field is given by

$$
\begin{aligned}
& P_{\text {out }}(\tau)=P_{\text {in }}(\tau) \exp \{h(\tau)\} \\
& \phi_{\text {out }}(\tau)=\phi_{\text {in }}(\tau)-\frac{1}{2} \alpha h(\tau)
\end{aligned}
$$

Here, $h$ is the SOA integrated gain, which is defined as

$$
h(\tau)=\int_{0}^{L} g(z, \tau) d z
$$


where $g$ is the SOA differential gain and $L$ is SOA length.

The integrated gain $h$, which depends on $\tau$, is given by the following equation;

$$
\frac{d h}{d \tau}=\frac{g_{0} L-h}{\tau_{c}}-\frac{P_{i n}(\tau)}{\tau_{c} P_{s}}\{\exp (h)-1\}
$$

Here, $g_{0}$ is the SOA small signal gain, $P_{i n}(\tau)$ is the input pulse power, and $P_{s}$ is the saturation power.

The chirp $\Delta v(\tau)$ is given by

$$
\Delta v(\tau)=-\frac{1}{2 \pi} \frac{\partial \phi}{\partial \tau}
$$

and by using (6) and (9), we obtain

$$
\Delta v_{\text {out }}(\tau)=\Delta v_{\text {in }}(\tau)+\frac{\alpha}{4 \pi} \frac{\partial h}{\partial \tau}
$$

where $v_{\text {in }}(\tau)$ and $v_{\text {out }}(\tau)$ are the input and output of the frequency chirp, respectively. These equations allow us to analyze the effect of the remaining downstream signal and the chirp characteristics.

\subsection{Calculated Result}

As described above, we have to consider two parameters that have an impact on the receiver sensitivity and transmission performance in the proposed system. These are i) the downstream signal ER, and ii) the upstream signal ER or modulation index of the injection current. i) involves a trade-off between the up- and downstream received sensitivities, because increasing the downstream signal ER improves the downstream receiver sensitivity, but it increases the remaining downstream pattern and reduces the upstream receiver sensitivity. ii) also involves a trade-off between back-to-back BER and transmission performance. This is because high extinction ratios increase the eye-opening and improve the back-to-back BER, but they generate high frequency chirp, which limits the transmission distance. Accordingly, we analyzed the effect of the up- and downstream signal ER by calculating the BER and frequency chirp.

\subsubsection{Effect of Remaining Downstream Signal}

First, we used Eq. (5) to calculate the effect of the remaining downstream signal. In this calculation, we set the bitrate of the up- and downstream signals at $1.25 \mathrm{Gbit} / \mathrm{s}$. The SOA specifications were set as follows; $3 \mathrm{~dB}$ saturated power of $0 \mathrm{dBm}$, a small signal gain of $15 \mathrm{~dB}$, and a carrier lifetime of $300 \mathrm{ps}$. In addition, we set the optical power at $3 \mathrm{dBm}$ at the SOA input and the upstream ER at its ideal value. As noted above, increasing the downstream ER reduces the saturation characteristic and strengthens the remaining pattern. As a result, increasing the downstream ER degrades the receiver sensitivity, so we analyzed the downstream ER dependence of the receiver sensitivity.

Figure 5 shows the received performance while changing the input signal ER. In Fig. 5, the solid line shows the

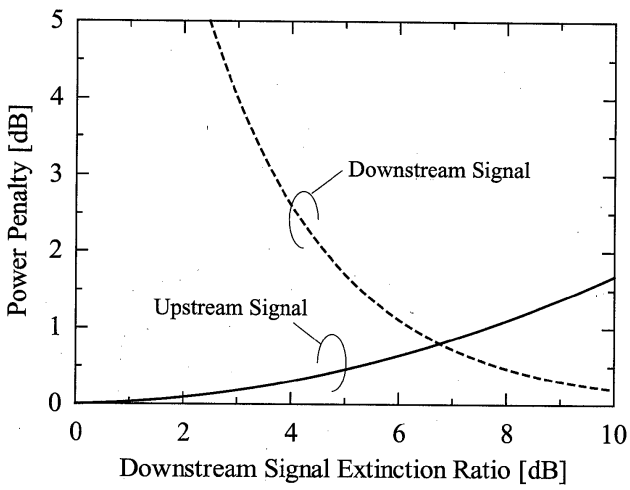

Fig. 5 Power penalty of up- and downstream signals calculated by changing the downstream signal ER. The solid and dotted lines show the power penalties of the up- and downstream signals, respectively.

power penalty of the upstream signal, and to show the tradeoff between the up- and downstream signal penalty, the power penalty of the downstream signal is given by the dotted line in the same figure. As can be seen, the upstream receiver sensitivity is degraded and the downstream receiver sensitivity is improved as the downstream ER is increased, so there is a trade-off between the downstream ER and the upstream receiver sensitivity. This figure shows that we must set the downstream ER at 5-9 dB to keep both up- and downstream power penalties below $1.5 \mathrm{~dB}$.

In addition, increasing the upstream ER reduces the effect of the remaining downstream signal pattern but also strengthens the frequency chirp, which limits the transmission distance. We analyze this chirp effect below.

\subsubsection{Effect of Frequency Chirp}

We calculated the optical output waveform and instantaneous optical frequency change of the saturated SOA by solving (10). Here, in the WCDR, there are two sources of frequency chirp. Because the chirp depends on the carrier density, it is generated when erasing the downstream signal and when modulating the upstream signal.

Figure 6 shows the erasing process and its attendant frequency shift. In this calculation, we set the bitrate and SOA specifications at the same values used in the previous calculation. Additionally, because the downstream signal can be amplified enough by the LA when receiving, the downstream ER was set at $5 \mathrm{~dB}$ where the power penalty of the upstream signal is smallest in the region of 5-9 dB ER which the penalty of bidirectional signals are enough small. In Fig. 6(a), the dashed line shows the optical waveform of the SOA input and the solid line shows that of the SOA output. It reveals that the SOA can erase the input signal. Figure 6(b) shows the optical frequency shift when the input signal chirp is 0 , and from this figure, the SOA yields an $8 \mathrm{GHz}$ frequency shift.

Next, we calculated the SOA modulation waveform and the frequency shift. Figure 7(a) shows the SOA output of the optical waveform generated by driving the injection 


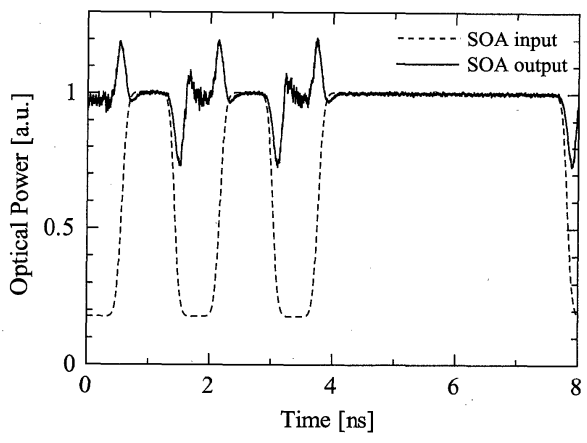

(a)

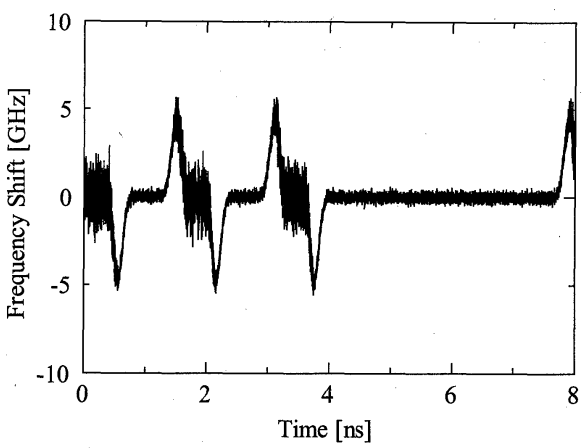

(b)

Fig. 6 Calculated (a) optical waveform and (b) optical frequency shift when erasing input data with the saturated SOA. In (a), the dotted and solid lines show the input and output waveforms, respectively.

current. In this figure, the SOA input is a continuous wave with an optical power of $3 \mathrm{dBm}$; all the SOA specifications are the same as in the previous calculation. When modulating the SOA injection current, the output optical power is modulated because of the change in the SOA gain, but at the same time, a frequency shift is generated, as shown in Fig. 7(b). Under these conditions, the output frequency shift is over $15 \mathrm{GHz}$. Since this shift exceeds that created by the erasing process, we can consider the transmission performance to be degraded more by the modulation process than by the erasing process.

Furthermore, we ran the same calculations for the data rewriting process, which includes the erasing and modulating processes. Figure 8 shows the calculated result; the conditions are the same as in the previous calculations. In Fig. 8(a), the solid line shows the input optical waveform and the dashed line shows the output waveform. We can see that the SOA can erase the input signal and generate a new signal simultaneously. Figure 8(b) shows the frequency shift imposed by the data rewriting process. Since this shift mirrors that created by the modulation process, these results confirm that the modulation process is a dominant chirp source.

Finally, the degradation in transmission performance was calculated as the power penalty. Figure 9 shows the transmission distance dependence of the power penalty. As shown in Fig. 9, the power penalty is less than $1 \mathrm{~dB}$ when the transmission distance increases to $40 \mathrm{~km}$ where the dispersion is $640 \mathrm{ps} / \mathrm{nm}$. A $40 \mathrm{~km}$ transmission is sufficient for

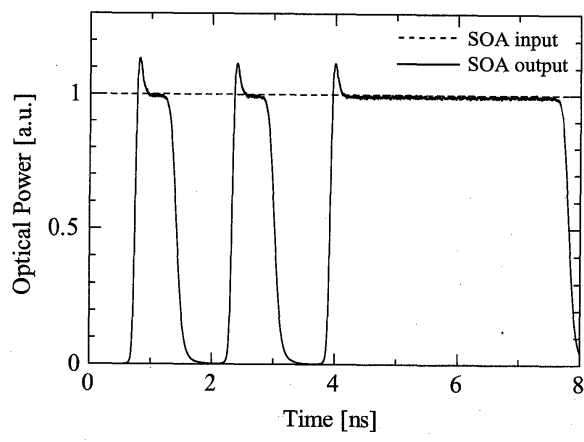

(a)

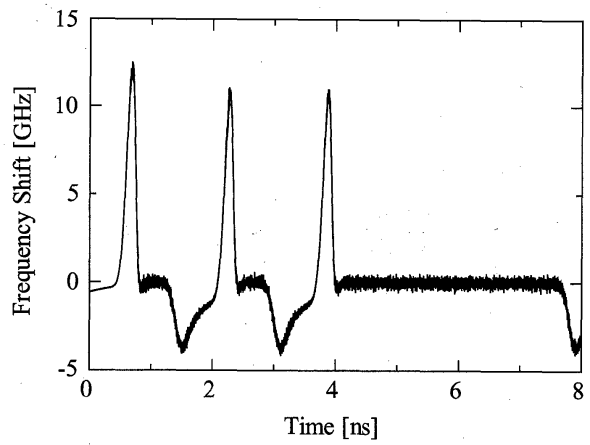

(b)

Fig. 7 Calculated (a) optical waveform and (b) optical frequency shift when modulating using the saturated SOA. In (a), the dotted and solid lines show the input and output waveforms, respectively.

most access networks, so this confirms that the WDM-PON using WCDR can realize access networks.

\subsubsection{SOA Frequency Dependence}

Finally, we analyzed the impact of the SOA frequency dependence. This frequency dependence was not considered in the previous calculations because the bitrate was only $1.25 \mathrm{Gbit} / \mathrm{s}$, but when the bitrate increases to between several Gbit/s and $10 \mathrm{Gbit} / \mathrm{s}$, the frequency dependence has a significant influence on receiver sensitivity. The reason for this is the SOA carrier lifetime which is typically of subnanosecond order.

We have to consider the bitrate of the up- and downstream signals. If the upstream bitrate increases, the SOA modulation speed cannot follow the upstream signal and the modulating signal eye pattern is degraded, which reduces the receiver sensitivity of the upstream signal. Consequently, it is difficult to increase the upstream bitrate above several Gbit/s, so we consider higher a downstream bitrate in this section.

The presence of the remnants of the downstream signal in the upstream signal is seen as another problem limiting the upstream bitrate, but surprisingly, increasing the downstream bitrate beyond the upstream bitrate alleviates both problems. This is because we can remove the high frequency remnants of the downstream signal from the upstream signal by using a low pass filter (LPF) in the upstream signal receiver. That is, placing a frequency filter 


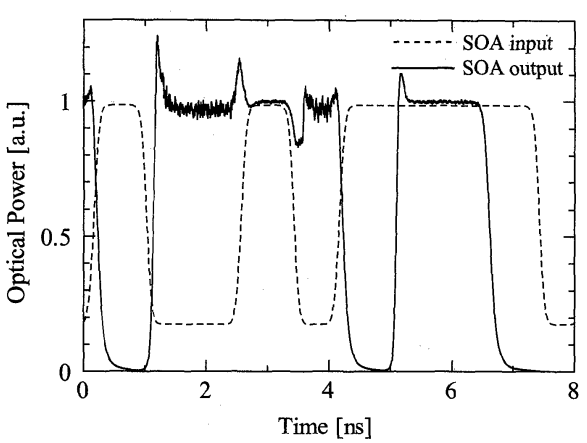

(a)

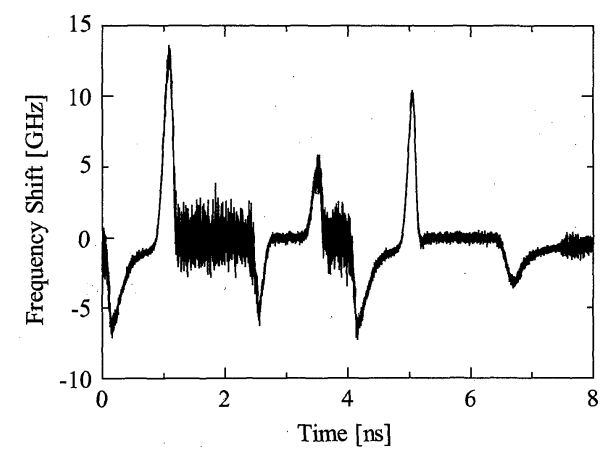

(b)

Fig. 8 Calculated (a) optical waveform and (b) optical frequency shift when rewriting input data with the saturated SOA. In (a), the dotted and solid lines show the input and output waveforms, respectively.

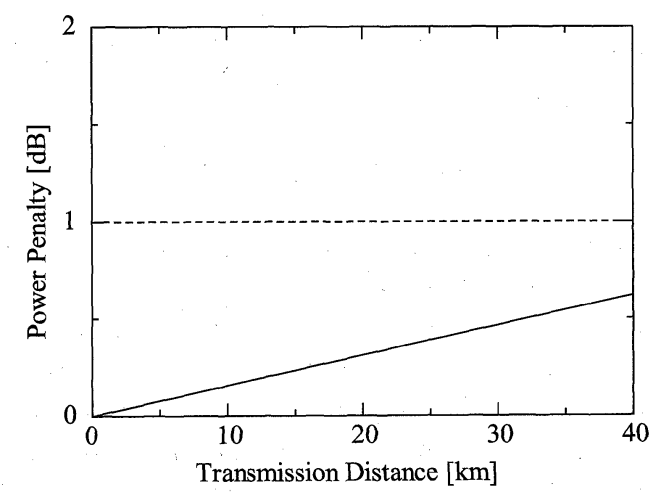

Fig. 9 Calculated transmission penalty at bidirectional $1.25 \mathrm{Gbit} / \mathrm{s}$.

in the upstream receiver offsets the poor SOA frequency response in the high frequency region. We verified this effect for downstream signal bitrates of up to $10 \mathrm{Gbit} / \mathrm{s}$. Figure 10 shows the power penalty imposed by the downstream signal bitrate. As can be seen, using a LPF (Bessel type, $3 \mathrm{~dB}$ bandwidth of $938 \mathrm{MHz}$ ) in the upstream receiver significantly improves the receiver performance, especially with very high speed downstream signals. Consequently, although the SOA's frequency response degrades the upstream receiver sensitivity because of the remaining high frequency component of the downstream signal, placing a LPF in the upstream receiver allows the downstream bitrate to be raised to $10 \mathrm{Gbit} / \mathrm{s}$.

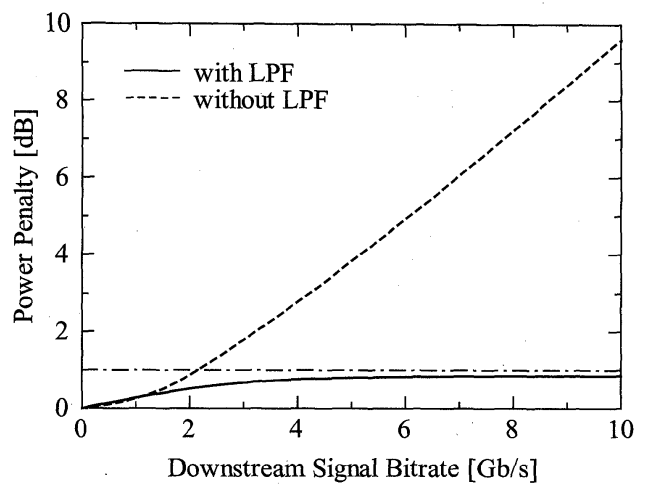

Fig. 10 Power penalty of upstream signal calculated by changing the downstream signal bitrate.

\section{Experiment}

We measured the frequency shift and the bit error performance of the WCDR. In this experiment, the WCDR consisted of an erbium doped fiber amplifier (EDFA) as the linear amplifier and a saturated SOA in order to measure only the characteristics of the saturated SOA; however, because the function of the EDFA is simply to amplify the downstream signal to the saturation power of SOA, we can reduce the cost by using another SOA instead of the EDFA with a monolithic integration of two SOAs.

Additionally, the saturated SOA had the following structural parameters; an active layer thickness of $0.4 \mu \mathrm{m}$, and optical waveguide width of $0.6 \mu \mathrm{m}$, an SOA length of $750 \mu \mathrm{m}$, a small signal gain of $15 \mathrm{~dB}$ and $3 \mathrm{~dB}$ saturation power of $0 \mathrm{dBm}[13]$.

First, we report the measured bit error performance before and after transmission for the same bitrate of $1.25 \mathrm{Gbit} / \mathrm{s}$ for both up- and downstream signals.

Then, we show the frequency response of the saturated SOA used in this experiment.

Finally, we describe the measured impact of raising the downstream signal.

\subsection{Transmission Experiment for Bidirectional 1.25 Gbit/s}

The experimental setup for measuring the bit error performance is shown in Fig. 11.

We used an external cavity laser as the light source, and set the wavelength at $1550 \mathrm{~nm}$. The output of the laser was modulated by an $\mathrm{LiNbO}_{3}$ external modulator with a $1.25 \mathrm{Gbit} / \mathrm{s} \mathrm{NRZ}$ signal [pseudo random binary sequence (PRBS) $\left.2^{7}-1\right]$. Here, we set the ER at $5 \mathrm{~dB}$ where the power penalty of downstream signal is $1.5 \mathrm{~dB}$ and upstream signal is $0.5 \mathrm{~dB}$ in the calculation. The optical signal was transmitted to the WCDR through single-mode fiber (SMF) and a variable attenuator. In the WCDR, the optical signal was amplified to the SOA saturation power by using the EDFA as a linear amplifier; the band pass filter (BPF) was set at $1550 \mathrm{~nm}$ to remove the EDFA amplified spontaneous 


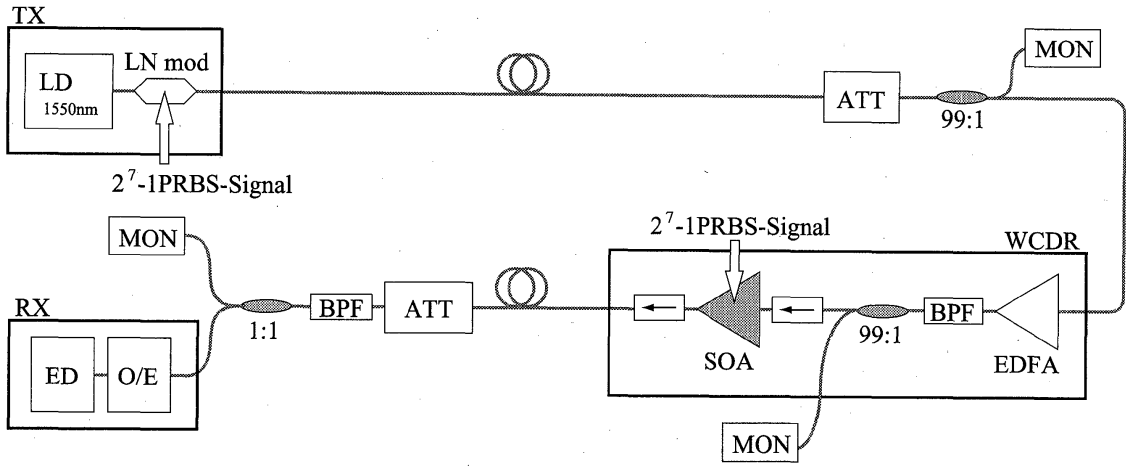

Fig. 11 Schematic diagram of experimental setup.
TX: Transmitter

RX: Receiver

LD: Laser Diode

LN mod: $\mathrm{LiNbO}_{3}$ Modulator

ATT: Attenuator

MON: Power Monitor

EDFA: Erbium Doped Fiber Amplifier

BPF: Band Pass Filter

SOA: Semiconductor Optical Amplifier

O/E: Optical / Electrical converter

ED: Error Detector (a)

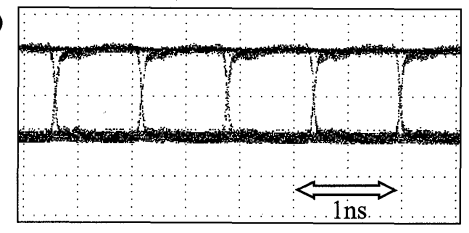

(b)

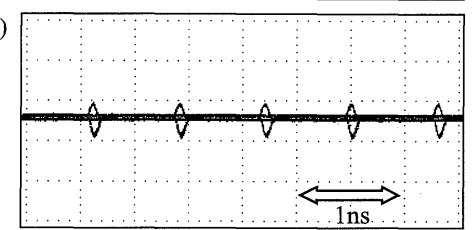

(c)

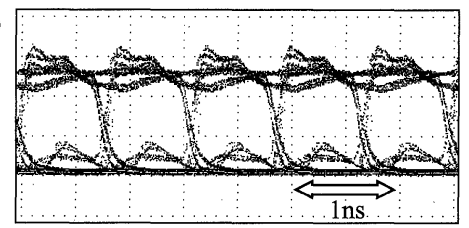

(d)

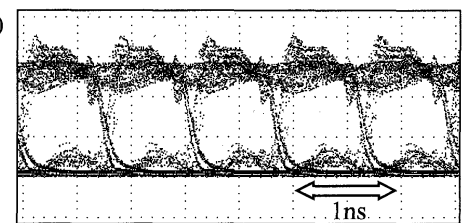

Fig. 12 Measured eye pattern; (a) modulated pattern before SOA, (b) after the SOA erasing process, (c) after the SOA modulation process when inputting a $\mathrm{CW}$ and (d) after the SOA rewriting process.

emission (ASE) noise. Next, the SOA erased the downstream signal through its saturation characteristic. At the same time, by driving the injection current of the SOA, the upstream signal was generated at $1.25 \mathrm{Gbit} / \mathrm{s}$ NRZ [PRBS $\left.2^{7}-1\right]$, and sent to the receiver. The WCDR was connected to the receiver by SMF transmission fiber and a variable attenuator, so chromatic dispersion was present in the upstream signal. Here, the dispersion penalty increases with the frequency shift or chirp. After transmission, the upstream signal was received by an optical receiver based on an avalanche photo diode (APD). The BER characteristic were measured with an error detector (ED). Figure 12 shows the eye diagrams obtained at (a) SOA input, (b) SOA output without current modulation (= data erasing), (c) SOA modulated response with a $\mathrm{CW}$ input, and (d) SOA output with new data modulation (= data rewriting). A transient re- sponse was present in (b), but the space level was amplified to the mark level. Therefore, by using the saturated SOA, we can erase the downstream signal and generate a continuous wave. (d) is the modulated lightwave with a new signal created by the rewriting process. Compared with (c), the mark level has some slight fluctuation, but clear eye opening can be obtained, so new data was generated. Figure 13 shows the frequency shift of the SOA output; (a) after erasing, (b) after modulation, (c) after rewriting. These figures show that each process also imposes some frequency shift, but erasing yields a smaller shift than modulation, because the extinction ratio of the downstream signal is lower than the upstream signal modulation. This can be seen from Fig. 13(c). In (c), the frequency shift is similar to (b), so when operating the SOA as a data rewriter, we can consider just the frequency chirp of the modulation process and ignore that of the erasing process. Figure 14 shows the bit-error performance of the WCDR for 0 to $40 \mathrm{~km}$ fiber transmission. As shown in Fig. 14, the power penalty after a $40 \mathrm{~km}$ transmission is less than $1 \mathrm{~dB}$; these results correspond to the calculated results. Since most access lines are less than $40 \mathrm{~km}$ long, we can say that installing a WCDR in a WDM-PON system makes it possible to realize a wavelength independent ONU.

\subsection{SOA Frequency Response}

Figure 15 shows the saturated SOA frequency response at various input optical powers. Here, we set the input optical extinction ratio at $5 \mathrm{~dB}$. As shown Fig. 15, the frequency response increases as the input frequency increases and the input optical power decreases. The dependence on the input optical power occurs because the SOA saturation characteristic is low at small input powers and high at high input powers. However, although the optical input power was sufficiently high, the frequency response increases as the frequency rises. This is because the frequency response depends on the SOA carrier lifetime, typical values of which are of sub-nanosecond order. From this result, we can say that the saturation characteristic is sufficient in the low frequency region, for example, for giga-bit ethernet $(\mathrm{GbE})$ communication.

Furthermore, when the downstream bitrate is higher 


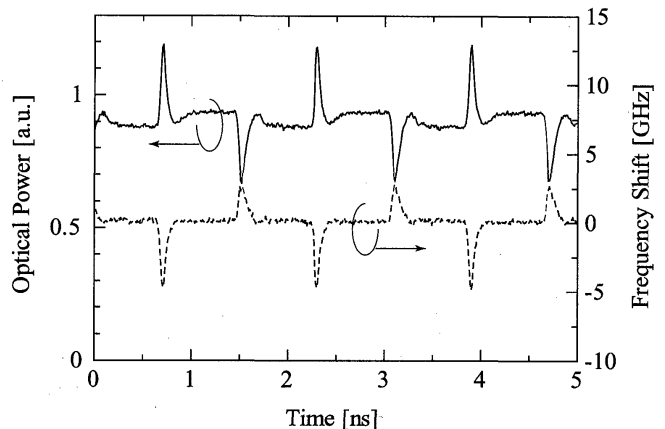

(a)

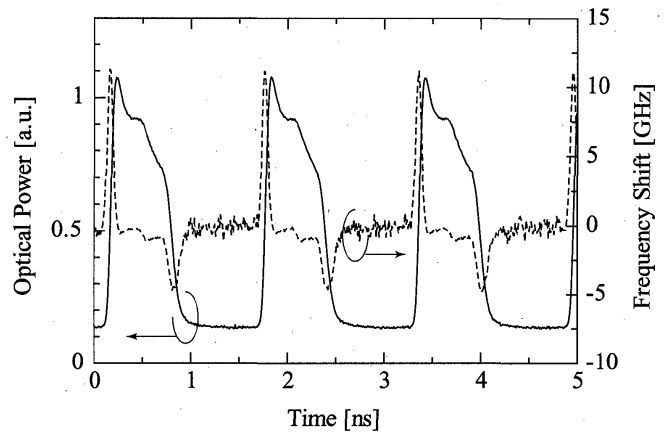

(b)

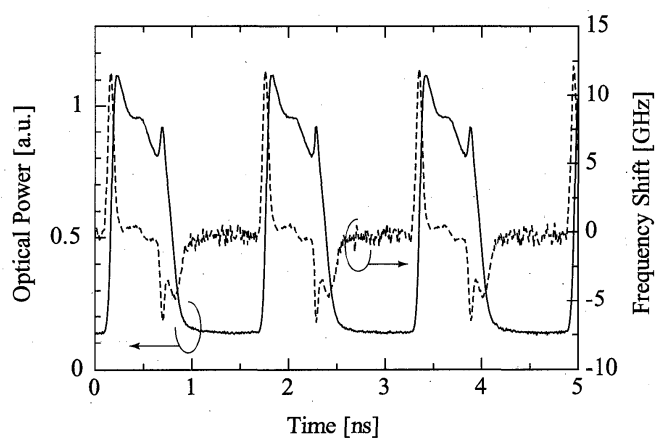

(c)

Fig. 13 Measured optical waveform and frequency shift; (a) after the SOA erasing process, (b) after the SOA modulation process when inputting $\mathrm{CW}$ and (c) after the SOA rewriting process.

than the upstream bitrate, we do not need to consider the frequency response in the high frequency region. In general, the high frequency noise is rejected by installing an LPF in the receiver that has a bandwidth of about 0.75 times the bitrate. The shaded area in Fig. 15 shows the receiver bandwidth when the upstream signal is $1.25 \mathrm{Gbit} / \mathrm{s}$. As can be seen, when the downstream bitrate increases, the high frequency noise from the remaining downstream signal lies outside the receiver bandwidth. As a result, the performance degradation of the upstream signal is minimized when the downstream signal increases. We provide a detailed explanation of this phenomenon in Sect. 5.3.

\subsection{Transmission Experiment for Asymmetric Transmis-} sion

When the bitrate of the downstream signal is higher than

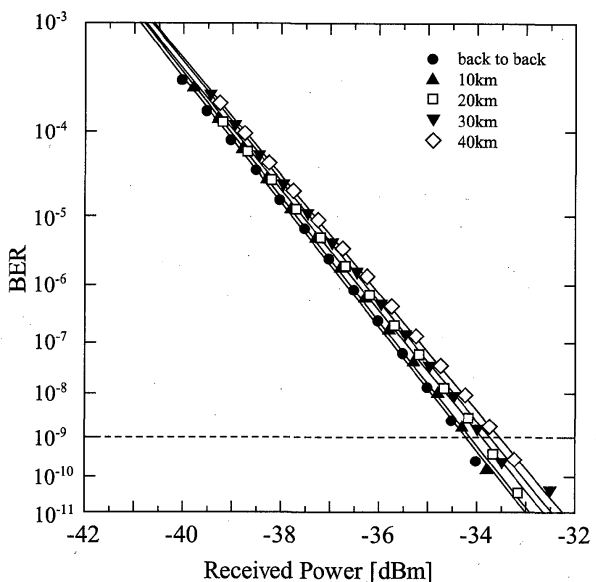

Fig. 14 Measured BER performance at $1.25 \mathrm{Gbit} / \mathrm{s}$ downstream and $1.25 \mathrm{Gbit} / \mathrm{s}$ upstream as a function of received optical power.

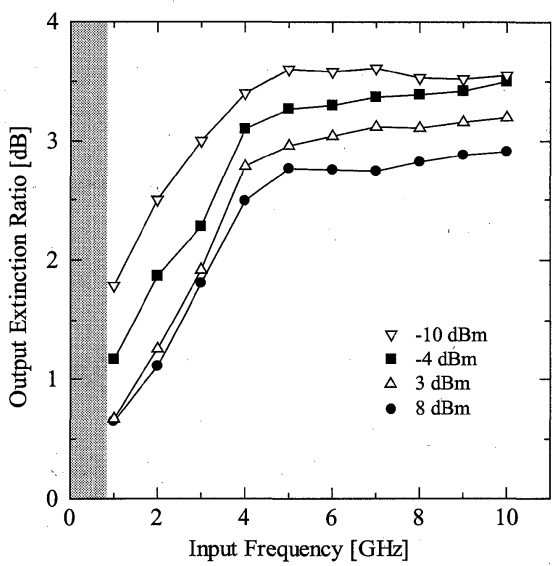

Fig. 15 Measured frequency response of saturated SOA as a parameter of SOA input power. Filled circles: $8 \mathrm{dBm}$ input power. Open triangles: $3 \mathrm{dBm}$. Filled squares: $-4 \mathrm{dBm}$. Open inverse triangles: $-10 \mathrm{dBm}$. Shaded area: $1.25 \mathrm{Gbit} / \mathrm{s}$ receiver bandwidth.

that of the upstream signal, for example more than several $\mathrm{Gbit} / \mathrm{s}$, the downstream signal cannot be erased completely because of the SOA carrier lifetime, as shown in Fig. 15. However, as described in Sect. 5.2, the use of a low pass filter at the upstream receiver overcomes this problem.

We measured the power penalty using the same setup as in the previous experiment. Here, we modulated the upstream signal at $1.25 \mathrm{Gbit} / \mathrm{s}$ with $2^{7}-1$ PRBS bit sequence; the downstream signal bitrate was set at $2.5 \mathrm{Gbit} / \mathrm{s}$ and $10 \mathrm{Gbit} / \mathrm{s}$. Other conditions were the same as in the experiment described in Sect. 5.1.

Figure 16 shows the results. Increasing the transmission distance increased the penalty caused by wavelength dispersion and frequency chirp, but the penalty did not depend on the downstream signal bitrate. This result shows that limiting the receiver bandwidth removes the unsuppressed downstream signal pattern sufficiently, so asymmetric bidirectional transmission can be realized.

Thus, we confirmed that the WCDR can realize a $40 \mathrm{~km}$ transmission with $10 \mathrm{Gbit} / \mathrm{s}$ downstream and $1.25 \mathrm{Gbit} / \mathrm{s}$ up- 


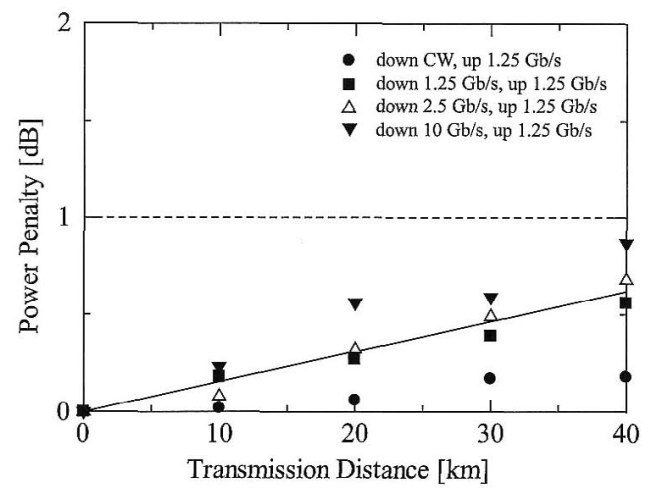

Fig. 16 Measured transmission penalty as a parameter of downstream signal bitrate. Filled circles: CW downstream and $1.25 \mathrm{Gbit} / \mathrm{s}$ upstream. Filled squares: $1.25 \mathrm{Gbit} / \mathrm{s}$ downstream and $1.25 \mathrm{Gbit} / \mathrm{s}$ upstream. Open triangles: $2.5 \mathrm{Gbit} / \mathrm{s}$ downstream and $1.25 \mathrm{Gbit} / \mathrm{s}$ upstream. Filled triangles: $10 \mathrm{Gbit} / \mathrm{s}$ downstream and $1.25 \mathrm{Gbit} / \mathrm{s}$ upstream.

stream signals.

\section{Conclusion}

We have proposed a data rewriting technique that allows us to realize a wavelength independent ONU, and also proposed a high capacity and low-cost WDM-PON access system that uses the wavelength channel data rewriter. We analyzed the chirp characteristic of the SOA and its dispersion penalty when using a saturated SOA to erase, modulate, and rewrite the upstream signal. This analysis revealed a penalty of less than $1 \mathrm{~dB}$ from back-to-back transmission, so costeffective WDM access networks are now possible.

We also described experiments on full-duplex bidirectional transmission with $1.25 \mathrm{Gbit} / \mathrm{s}$ data rates. The experimental and calculated results were good agreement (less than $1 \mathrm{~dB}$ penalty), so effective communication is possible over $40 \mathrm{~km}$ of SMF. Moreover, we showed the possibility that the futher cost reduction can be acheived by using two integrated SOA.

In addition, we analyzed and demonstrated the benefit of raising the downstream signal speed to $10 \mathrm{Gbit} / \mathrm{s}$ while keeping the upstream signal bitrate at $1.25 \mathrm{Gbit} / \mathrm{s}$. This speed differential allows the remnant of the downstream signal in the upstream signal to be stripped out by installing a low pass filter in the upstream signal receiver; the dispersion penalty was also less than $1 \mathrm{~dB}$, so this technique is very practical.

\section{Acknowledgments}

We thank Dr. Hiroki Takesue [8] of NTT Basic Research Laboratories, NTT Corporation and Dr. Naoto Yoshimoto [13] of NTT Access Network Service Systems Laboratories, NTT Corporation.

\section{References}

[1] R.D. Feldman, E.E. Harstead, S. Jiang, T.H. Wood, and M. Zirngibl,
"An evaluation of architectures incorporating wavelength division multiplexing for broad-band fiber access," J. Lightwave Technol., vol.16, no.9, pp.1546-1559, Sept. 1998.

[2] N.J. Frigo, P.P. Iannone, P.D. Magill, T.E. Darcie, M.M. Downs, B.N. Desai, U. Koren, T.L. Koch, C. Dragone, H.M. Presby, and G.E. Bodeep, "A wavelength-division multiplexed passive optical network with cost-shared components," IEEE Photonics Technol. Lett., vol.6, no.11, pp.1365-1367, Nov. 1994.

[3] T. Yoshida, S. Kimura, and K. Kumozaki, "A novel backreflection suppression method using phase modulation for $10 \mathrm{Gbit} / \mathrm{s}$ singlefiber WDM loopback networks," Proc. 30th European Conf. on Opt. Commun., no.Tu3.6.6, pp.226-227, Stockholm, Sweden, Sept. 2004.

[4] H. Nakamura, H. Suzuki, J. Kani, and K. Iwatsuki, "A widearea carrier-distributed WDM-based access network accommodating GbE and $10 \mathrm{GbE}$ services," Proc. Opt. Fiber Commun., no.OFA4, Anaheim, California, USA, March 2005.

[5] W. Hung, C.K. Chan, L.K. Chen, and C. Lin, "System characterization of a robust re-modulation scheme with DPSK downstream traffic in a WDM access network," Proc. 29th European Conf. on Opt. Commun., no.We3.4.5, pp.514-515, Rimini, Italy, Sept. 2003.

[6] W. Hung, C.K. Chan, L.K. Chen, and F. Tong, "An optical network unit for WDM access networks with downstream DPSK and upstream re-modulated OOK data using injection-locked FP laser," Proc. Opt. Fiber Commun., no.TuR2, Atlanta, Georgia, USA, March 2003.

[7] G.W. Lu, N. Deng, C.K. Chan, and L.K. Chen, "Use of downstream inverse-RZ signal for upstream data re-modulation in a WDM passive optical network," Proc. Opt. Fiber Commun., no.OFI8, Anaheim, California, USA, March 2005.

[8] H. Takesue and T. Sugie, "Wavelength channel data rewrite using saturated SOA modulator for WDM networks with centralized light sources," J. Lightwave Technol., vol.21, no.11, pp.2546-2556, Nov. 2003.

[9] G.P. Agrawal and N.A. Olsson, "Self-phase modulation and spectral broadening of optical pulses in semiconductor laser amplifiers," IEEE J. Quantum Electron., vol.25, no.11, pp.2297-2306, Nov. 1989.

[10] T. Watanabe, N. Sakaida, H. Yasaka, F. Kano, and M. Koga, "Transmission performance of chirp-controlled signal by using semiconductor optical amplifier," J. Lightwave Technol., vol.18, no.8, pp.1069-1077, Aug. 2000.

[11] C.H. Henry, "Theory of the linewidth of semiconductor lasers," IEEE J. Quantum Electron., vol.18, no.2, pp.259-264, Feb. 1982.

[12] M. Osinski and J. Buus, "Linewidth broadening factor in semiconductor lasers - An overview," IEEE J. Quantum Electron., vol.23, no.1, pp.9-29, Jan. 1987.

[13] N. Yoshimoto, T. Ito, K. Magari, Y. Kawaguchi, K. Kishi, Y. Kondo, Y. Kadota, O. Mitomi, Y. Yoshikuni, Y. Hasumi, Y. Tohmori, and O. Nakajima, "Four-channel polarization-insensitive SOA gate array integrated with butt-jointed spot-size converters," Electron. Lett., vol.33, no.11, pp.2045-2046, Nov. 1997.

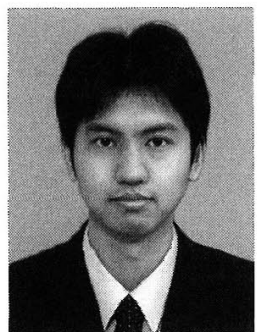

Satoshi Narikawa received the B.E. and M.E. degrees in electrical and clectronic engineering from Tokyo Institute of Technology, Tokyo, Japan, in 2001 and 2003, respectively. In 2003, he joined the NTT Access Network Service Systems Laboratories, Chiba, Japan, where he has engaged in research on innovative optical multiplexing systems for access applications. 


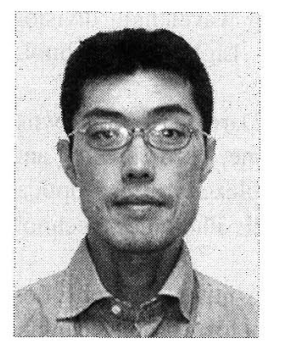

Hiroaki Sanjoh received the B.E. and M.E. degrees from Hokkaido University, in 1990, 1992, respectively. In 1992 he joined NTT opto-electronics laboratory and since then he has been engaged in reserch and development of semiconductor optical devices and optical transmission systems.

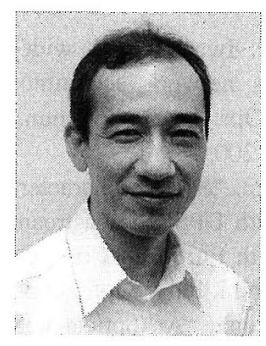

Naoya Sakurai received the B.E. degree in applied electronics engineering from the University of Electro-Communications, Japan, in 1981. In 1981 he joined NTT Laboratories and has been engaged in research on the highdefinition television transmission systems. His current interests are in development of optical video transmission systems. He is now a Senior Research Engineer in the Optical Access Innovation Project, NTT Access Network Service Systems Laboratories, Chiba, Japan.

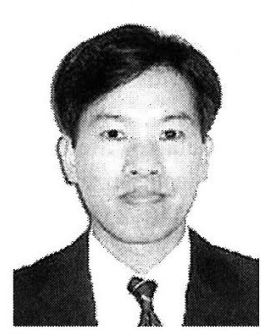

Kiyomi Kumozaki received the B.E. and M.E. degrees in electrical and electronics engineering from Toyohashi University of Technology, Toyohashi, Japan, in 1980 and 1982, respectively, and Ph.D degree from Tohoku University, Sendai, Japan, in 2000. In 1982, he joined the NTT Yokosuka Electrical Communication Laboratory, Kanagawa, Japan, where he was engaged in research on metallic digital transmission, and development of metallic digital access systems. From 1987 to 1990 , he worked on the development of ISDN basic rate access systems at the NTT Network Systems Development Center, Kanagawa, Japan. From 1991 to 1997, he was at the NTT Transmission Systems Laboratories, Kanagawa, Japan, where he carried out the research on passive optical access network for providing data communication, telephony, and video distribution services. From 1998 to 2000, he worked for NTT Communications Corporations, Tokyo, Japan, where he was engaged in building large scale LAN, WAN, and global network infrastructure for business enterprise. Since 2001, he has been with the NTT Access Service Systems Laboratories, Chiba, Japan, and working on research and development of nextgeneration access network including high speed passive optical network. Dr. Kumozaki is a member of the Institute of Electrical and Electronics Engineers (IEEE). 